\title{
Essais Lefranc pour la mesure de la perméabilité in situ : étude théorique et interprétation pratique
}

A. DHOUIB

SOLEN-Études, 48-50, rue Eugénie-

Le Guillermic, 92290 Villeneuve-le-Roi

\section{SHAHROUR}

Z. LAFHAJ

Laboratoire de Mécanique des Solides (URA 1441),

École centrale de Lille,

Cité scientifique, BP 48,

59651 Villeneuve-d'Ascq

Cedex

A. DELFAUT

LROP, Trappes
On étudie l'essai d'eau ponctuel connu en France sous le nom d'essai Lefranc pour la mesure in situ de la perméabilité du sol. On décrit les tendances actuelles, les sources d'erreur et les dispositions pratiques pour une exécution soignée de l'essai. On développe les équations qui régissent l'écoulement en rẻgime transitoire et on présente succinctement les valeurs du facteur de forme $m$ proposées par plusieurs auteurs. Puis, on exploite quatre essais réels, effectués à charge variable sur des sols de perméabilité différente, afin de montrer l'incidence des méthodes d'interprétation sur les valeurs du coefficient de perméabilité Lefranc. Face aux problèmes d'exécution, aux divergences des méthodes de calcul du facteur de forme et aux différences des résultats d'interprétation, on propose dans cette étude quelques $\alpha$ recommandations» pratiques permettant de réduire les erreurs affectant, sur le plan expérimental et d'interprétation, le calcul du coefficient de perméabilité.

\section{Lefranc permeability test : theoretical analysis and practical interpretation}

The a Lefranc test $x$ is a permeability test carried out in situ. The current trends and sources of error in this field tests are described and practical rules are submitted. The basic equations governing the transient state are reviewed and the shape factor values proposed by several authors are succinctly analyzed. Field tests results obtained from four variable head tests are compared in order to show the influence of the interpretative methods on hydraulic conductivity. With regard to execution problems, to the diverging m-values and to the differences between the interpreted results, practical " recommandations are suggested to permit to reduce errors in these field tests and their interpretation. 


\section{NOTATIONS}

D : diamètre de la cavité.

$\mathrm{h}(\mathrm{t})$ : charge hydraulique.

$\underline{\mathrm{dh}}$ : vitesse apparente de remontée ou de descente d'eau dans le tubage.

$\mathrm{h}_{\mathrm{a}} \quad$ : charge hydraulique à l'origine de la droite $\mathrm{h}(\mathrm{t})$ en fonction de $\mathrm{dh} / \mathrm{dt}$.

$h_{0}$ : charge hydraulique initiale.

$h_{p}$ : charge hydraulique correspondant au régime permanent.

$\mathrm{H}_{w} \quad$ : distance de la surface de la nappe au milieu de la cavité.

$\mathrm{H} \quad$ : distance du toit du substratum imperméable au milieu de la cavité.

$k_{1} \quad$ : coefficient de perméabilité Lefranc (en $\mathrm{m} / \mathrm{s})$.

L : longueur de la cavité.

m : facteur de forme de la cavité.

$\mathrm{m}_{\mathrm{o}}$ : facteur de forme pour une cavité éloignée des limites de l'aquifère $(m=m)$.

$\mathrm{Q}(\mathrm{t})$ : débit traversant la paroi de la cavité à un instant t donné.

$Q_{a} \quad$ : débit d'apport constant (débit injecté : $Q_{u} \neq 0$, débit absorbé : $Q_{\mathrm{a}}=0$ ).

$\mathrm{R}$ : extension latérale du domaine d'écoulement.

S : aire intérieure de la section droite horizontale du tubage.

$\alpha_{1} \quad$ : pente de la droite d'approximation de la forme exponentielle (lorsque $\mathrm{k}_{\mathrm{L}}$ est très faible).

$\alpha_{2} \quad$ : pente de la droite en coordonnées semilogarithmiques.

$\alpha_{3} \quad$ : pente de la droite $\mathrm{h}(\mathrm{t})$ en fonction de $\mathrm{dh} / \mathrm{dt}$.

$\delta \mathrm{h}:$ abaissement du niveau d'eau dans le tubage (essai d'absorption).

$\Delta \mathrm{h} \quad$ : variation de la charge hydraulique pendant une variation du temps $\Delta t$ donnée.

\section{Introduction}

Les problèmes créés par l'eau dans le sol sont fondamentaux dans les projets de Génie civil. Les excavations profondes, le creusement de tunnel et les décaissements pour fouilles de sous-sols se multiplient. En zones urbaines ou rurales, les projets de décharges et de centres d'enfouissement nécessitent la protection des nappes et le respect des aspects écologiques. L'orjgine de ces problèmes et leur solution sont complexes.

Depuis longtemps, on sait déterminer le coefficient de perméabilité du sol en laboratoire sur des échantillons prélevés et considérés comme « intacts ». Les essais sont effectués dans des conditions idéales de percolation suivant l'axe de l'éprouvette. La perméabilité, appréciée suivant un chemin d'écoulement privilégié, est globale vis-àvis de I'échantillon, mais ponctuelle vis-à-vis du massif de sol à reconnaître; ceci la rend généralement peu représentative de l'ensemble du milieu le plus souvent hétérogène et anisotrope. D'une manière générale, le coefficient de perméabilité exprimé en $\mathrm{m} / \mathrm{s}$ n'est connu qu'à une puissance de 10 près. Enfin, les stratifications horizontales peuvent encore réduire considérablement ce coefficient d'une puissance de 10, voire de 100. Le fascicule 62, Titre V (MELT, 1993) précise clairement que les coefficients de perméabilité mesurés en laboratoire (norme NF P 94-090, AFNOR, 1992) ne doivent « servir qu'à la détermination du coefficient de consolidation $\mathrm{c}_{\mathrm{c}}$ et qu'il serait illusoire de les utiliser pour effectuer, entre autres, des calculs de débits de pompage ou d'exhaure ».

Avec le développement des moyens de mesure et d'essais, on assiste à un large essor des « essais d'eau ») en place pour le calcul du coefficient de perméabilité locale. Ces essais consistent à créer une cavité libre le long ou à la base d'un forage pour y générer une variation, dans le temps, de la charge hydraulique. L'essai de pompage est le plus représentatif car il permet de couvrir un volume de sol autour du puits de pompage suffisant pour intégrer les conditions réelles d'alimentation de la nappe. Mais, l'essai se heurte à des difficultés multiples en particulier en site urbain où les opérations de pompage peuvent entraîner les fines conduisant parfois à des tassements importants des mitoyens et des structures existantes. L'essai d'eau, connu en France sous le nom d'essai Lefranc (Lefranc, 1936, 1937), constitue à ce jour une solution intermédiaire car il permet de s'affranchir des problèmes de ravinement et de boulance. En plus, il s'applique à tous les sols sous la nappe et il est relativement rapide et peu coûteux. Cependant, sa validité reste liée aux conditions expérimentales souvent sensibles (Milligan, 1975 ; SCG, 1985) et parfois trompeuses (phénomènes de colmatage, mauvaise isolation de la cavité, communication de nappes, suivi peu précis de la variation de la charge hydraulique, arrêt hâtif de l'essai avant stabilisation). A cela s'ajoute le caractère ponctuel de la mesure car l'écoulement n'intéresse qu'un volume restreint de sol autour de la cavité. C'est pour cette raison que la norme NF P 94-132 (1992), propre à cet essai, stipule qu'il « ne doit pas être utilisé seul pour dimensionner un rabattement important de nappe n. De plus, la détermination du coefficient de perméabilité dans l'essai Lefranc dépend des dìmensions de la cavité et de la position de l'aquifère exprimé par la valeur du facteur de forme dont l'estimation est, sur le plan pratique, difficile en particulier dans les sols stratifiés (Cassan, 1979).

Dans cette communication, on met l'accent sur l'incidence des difficultés expérimentales, des divergences des approches du calcul du facteur de forme m et des sensibilités des méthodes d'interprétation sur le coefficient de perméabilité mesuré par l'essai Lefranc. On montre, en particulier, que lorsque l'essai est mal exécuté (mauvaise isolation de la cavité, phénomène de colmatage), les disparités dans l'estimation du coefficient de perméabilité peuvent être très importantes.

2

\section{Analyse expérimentale de l'essai Lefranc}

\section{1}

\section{Variantes de l'essai. Tendances actuelles}

L'essai Lefranc (Norme NF P 94-132, 1992) consiste à créer une cavité dans le sol et de générer une variation 
de la charge hydraulique $\mathrm{h}$ mesurée en fonction du temps (Fig. 1). La tendance actuelle en France est de procéder à des essais à charge constante ou à charge variable.

Dans le premier cas, la hauteur d'eau est stabilisée dans le forage (sols perméables). La durée de l'essai peut être, selon les cas, d'une à deux heures. Dans le cas des matériaux très perméables (sables et graviers, graves, remblais de décharge), il est très difficile sur le plan pratique de garder une charge constante pendant une durée suffisante. Lorsque le sol est moyennement perméable, il est possible de procéder aussi à l'exécution de l'essai à charge variable avec des intervalles de temps de mesure faibles allant de 30 secondes à 2 minutes.

Dans le second cas, on mesure la descente ou la remontée d'eau dans le forage. Dans les sols fins et peu perméables (argiles et marnes fermes, calcaire non fissuré, craie saine), on procède plutôt à des essais d'absorption; l'essai peut durer plusieurs heures à quelques dizaines d'heures. Le suivi de variation de la charge en fonction du temps se fait à l'aide d'un capteur de pression de haute précision muni d'un appareil d'enregistrement automatique de type MADOPLUS (IRIS-BRGM) avec des intervalles de temps de mesure qui peuvent aller de 2 à 10 minutes.

\section{2}

\section{Sources d'erreur et dispositions particulières}

Sur le plan expérimental, l'essai Lefranc nécessite des dispositions particulières car les sources d'erreur sont multiples. Hvorslev (1951), Gibson (1963, 1966), Wilkinson (1968), Weber (1968) et Bjerrum et al. (1972): distinguent quatre causes principales d'erreur propres aux essais d'eau dont trois sont applicables à l'essai

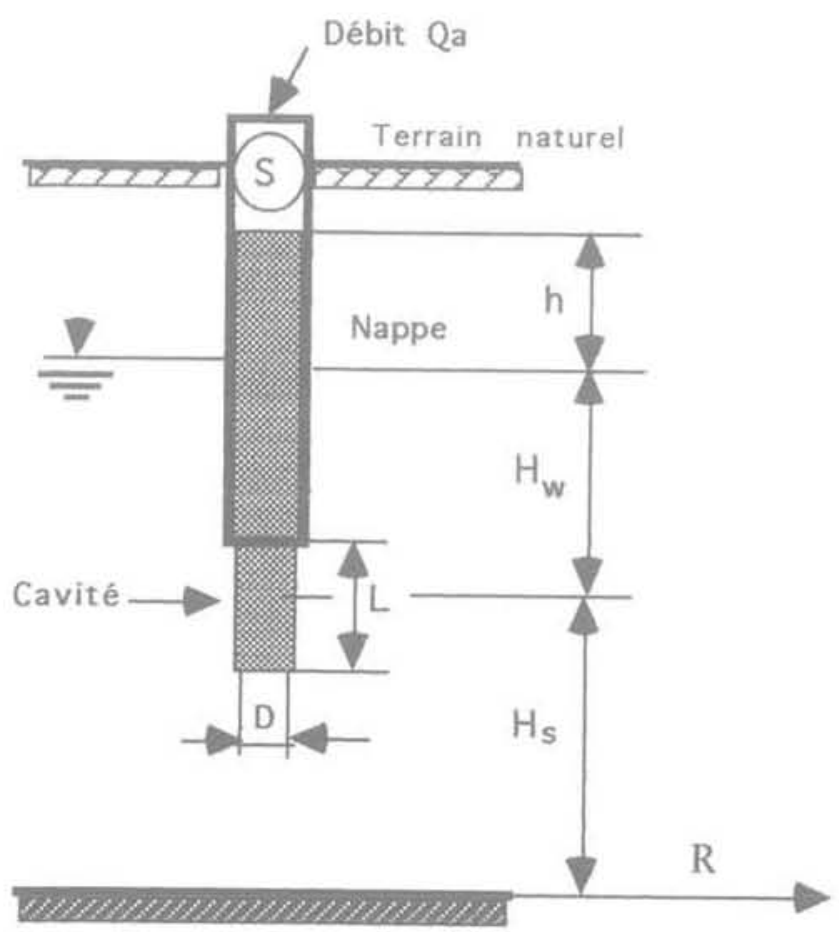

Substratum imperméable

FIG.1 Essais Lefranc: principe et notations. Lefranc test : principle and symbols.
Lefranc: mauvaise isolation de la lanterne, instabilité des parois de la cavité et phénomène de colmatage préalable ou en cours d'essai.

La norme NF P 94-132 décrit clairement les techniques de réalisation de la cavité en fonction du sol rencontré, mais il convient de préciser certains points pratiques acquis à travers l'exécution de quelques centaines d'essais afin de limiter les sources d'erreur : - la position de la nappe doit être connue avant la réalisation de l'essai car l'exploitation de l'essai n'est possible que lorsqu'il est effectué sous la nappe; en plus, l'estimation de $k_{1}$ nécessite une connaissance précise de la charge hydraulique appliquée (Chapuis, 1989); - le forage doit être réalisé à l'air ou à l'eau claire ; l'emploi d'une boue de forage est à proscrire car il conduit inévitablement à un colmatage préalable de la cavité ;

- pour un sol homogène (Fig. 2a), l'isolation de la cavité peut être assurée par un obturateur gonflable lorsque le sol est cohérent, mais la mise en place d'un tubage pour maintenir stables les parois de forage est indispensable dans les sols pulvérulents ;

- lorsque l'essai est effectué dans un sol reposant sous plusieurs couches de perméabilités différentes (sol stratifié, Fig. 2b), la cavité doit être réalisée avec un diamètre plus faible que le diamètre intérieur du tubage dont le diamètre extérieur doit être suffisamment grand afin d'assurer l'isolation complète de la cavité et d'éliminer les êventuelles fuites ;

- après la mise en place du tubage, la lanterne doit ètre curée au carottier de même diamètre que la cavité pour éliminer les débris dus au grattage. En plus, elle doit être soufflée à l'air sous pression pour éviter l'expansion des particules fines qui favorisent le colmatage, à condition que cette opération ne déstabilise la cavité (cas des sables) ;

- afin d'assurer la stabilité de la cavité, celle-ci doit être remplie par un gravier-filtre reposant sur un bouchon étanche (en argile ou en sobranite) ou un obturateur dilatable dans le cas des sols pulvérulents. L'emploi du filtre peu être aussi indispensable dans les sols cohérents comme les argiles vertes du Sannoisien ou les marnes bleues d'Argenteuil du Ludien où nous avons constaté dans certains cas un rétrécissement, voire une fermeture partielle de la cavité sous l'effet d'un gonflement parfois instantané.

\section{3}

Analyse théorique de l'essai Lefrane 3.1

\section{Équations générales}

A partir de la loi de Darcy, Hvorslev (1951) a montré que le débit $\mathrm{Q}(\mathrm{t})$ percolant à travers la surface de la cavité est, à un instant $t$ donné, proportionnel à la charge hydraulique $\mathrm{h}(\mathrm{t})$, au diamètre $\mathrm{D}$ de la cavité et au coefficient de perméabilité Lefranc $\mathrm{k}_{1}$. Le coefficient de proportionnalité est le facteur de forme m qui dépend (Fig. 3) des dimensions relatives de la cavité L/D (Hvorslev, 1951; Schneebeli, 1954, 1966), de la position de la nappe $\left(\mathrm{H}_{\mathrm{w}}\right)$ et du substratum imperméable $\left(\mathrm{H}_{\mathrm{s}}\right)$; la forme générale du débit $\mathrm{Q}(\mathrm{t})$, révisée par 


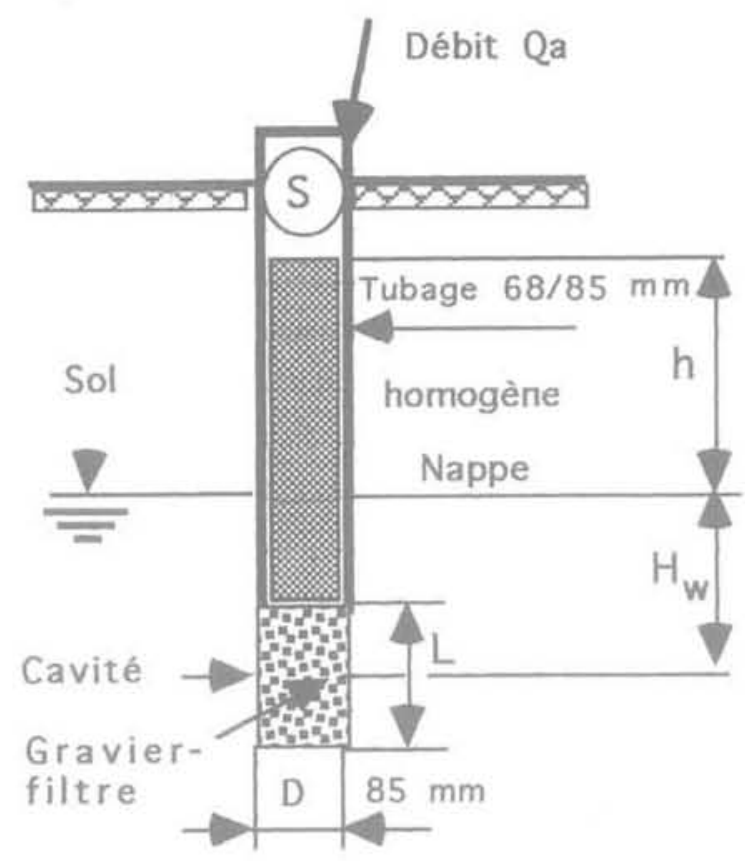

a

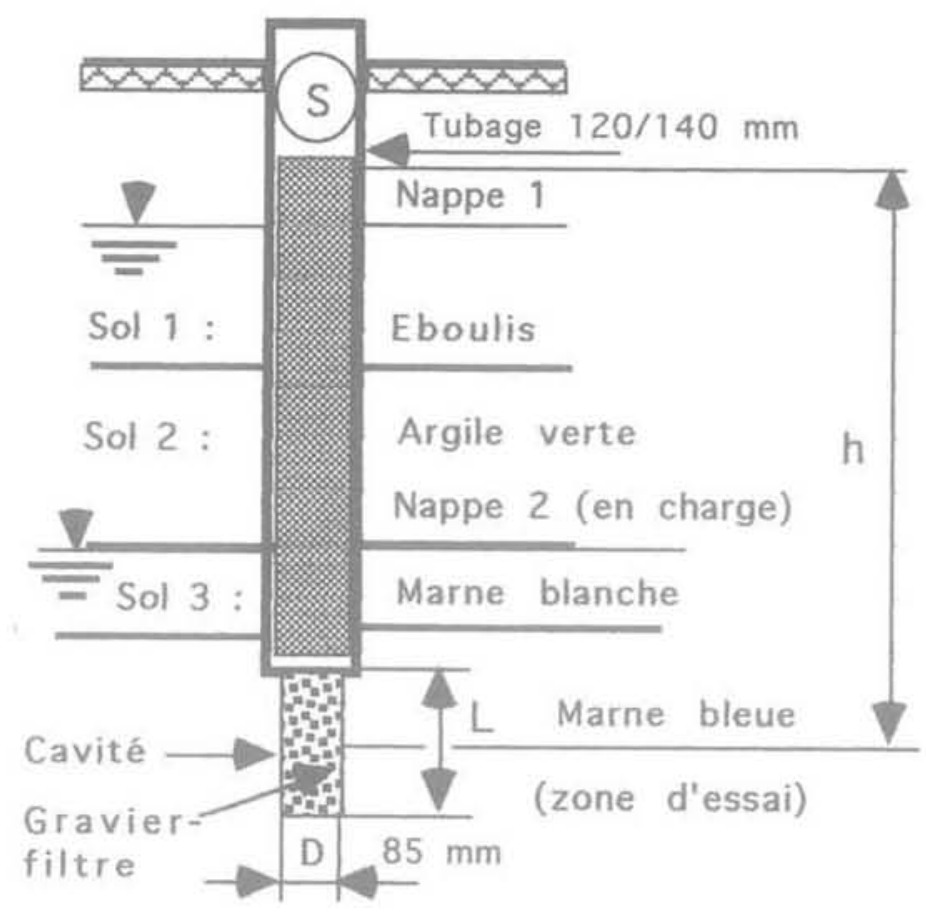

b

F1G.2 Dispositions particulières pour la réalisation des essais Lefranc. A Lefranc test configuration.

Boersma (1965) puis Bouwer et Jackson (1974) est :

$$
\mathrm{Q}(\mathrm{t})=\mathrm{m} \cdot \mathrm{k}_{\mathrm{L}} \cdot \mathrm{D} \cdot \mathrm{h}(\mathrm{t})
$$

En respectant les notations de la figure 1, la charge hydraulique h en régime transitoire est régie, sous un débit de pompage ou d'ínjection $\mathrm{Q}$, par l'équation de conservation de la masse :

$$
\mathrm{s} \cdot \frac{\mathrm{dh}}{\mathrm{dt}}+\mathrm{m} \cdot \mathrm{k}_{\mathrm{L}} \cdot \mathrm{D} \cdot \mathrm{h}=\mathrm{Q}_{\mathrm{n}},
$$

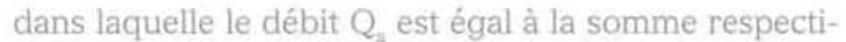
vement du débit dû à la variation du volume d'eau dans la colonne et du débit de filtration dans la cavité, avec S étant la section horizontale de la colonne d'eau (forage ou tubage).

L'équation (2) est une équation différentielle à variables séparées que l'on peut écrire:

$$
\mathrm{dt}=\frac{\mathrm{dh}}{\frac{\mathrm{Qa}}{\mathrm{S}}-\frac{\mathrm{m} \cdot \mathrm{k}_{\mathrm{L}} \cdot \mathrm{D}}{\mathrm{S}} \cdot \mathrm{h}}
$$
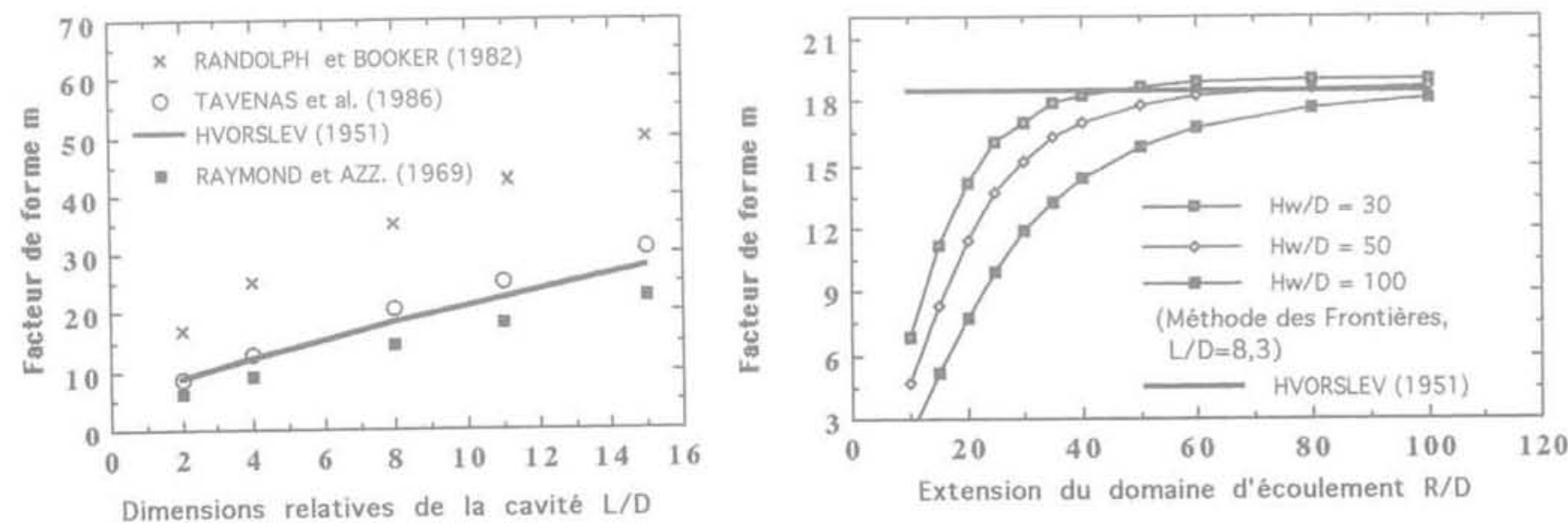

FIQ.3 Variation du facteur de forme $\mathrm{m}$ : a) incidence des dimensions de la cavité (différentes méthodes) : b) incidence de l'extension du domaine (méthode des Frontières, $L / D=8,3$ ).

Shape factor $\mathrm{m}: \mathrm{a}$ ) effect of aspect ratio L/B (various methods) ; b) effect of domain extension R/D (boundary elements method). 
Avec les conditions initiales : $(t=t, h=h)$ et dans le cas où $Q_{\mathrm{n}}$ est constant, l'intégration dé (3) est immédiate et on obtient la solution sous forme exponentielle qui régit l'écoulement, soit :

$$
h(t)=h_{p}-\left(h_{p}-h_{0}\right) \cdot e-\frac{m \cdot k_{L} \cdot D}{S} \cdot t
$$

où

$$
\mathrm{h}_{\mathrm{p}}=\frac{\mathrm{Q}_{\mathrm{a}}}{\mathrm{m} \cdot \mathrm{k}_{\mathrm{L}} \cdot \mathrm{D}}
$$

désigne la charge hydraulique en régime permanent (h tend vers $h_{\text {p }}$ lorsque t tend vers l'infini). Lorsque l'essai est effectué sans apport de débit $\left(\mathrm{Q}_{\mathrm{a}}=0\right)$, on obtient :

$$
h(t)=h_{0} \cdot e-\frac{m \cdot k_{L} \cdot D}{S} \cdot t
$$

ou encore :

$$
t=-\frac{S}{m \cdot k_{1} \cdot D} \operatorname{Ln} \frac{h(t)}{h o}
$$

Ces équations montrent que le coefficient de perméabilité est directement lié au facteur de forme dont la détermination est, sur le plan pratique, délicate. Nous allons ci-après présenter brièvement les méthodes proposées pour le calcul de ce facteur.

\section{2}

\section{Facteur de forme. État actuel des connaissances}

Plusieurs auteurs ont cherché à calculer le facteur de forme. En se basant sur les travaux de Dachler (1936), Hvorslev (1951) résout analytiquement l'équation de Laplace pour calculer le facteur de forme. Les auteurs comparent généralement leurs résultats aux valeurs de $m$ ainsi obtenues. Ces valeurs sont les plus utilisées en pratique courante avec m variant entre 10 et 30 pour les rapports courants de I/D allant de 2 à 15 comme le montre la figure 3 a où l'on présente, pour quatre auteurs, la variation du facteur de forme en fonction des dimensions relatives de la cavité considérée éloignée des limites de l'aquifère $(\mathrm{m}=\mathrm{m}$, Norme NF P 94-132, 1992). Il ressort de cette variation que m croît avec le rapport L/D. Les différences entre les valeurs de m sont à la fois inhérentes aux approximations des méthodes numériques et liées aux conditions aux limites et au choix du domaine (Chapuis, 1989). En effet, le choix du domaine physique se pose en particulier dans l'analyse numérique du facteur de forme par la méthode des éléments finis (Tavenas et al., 1986a, 1986 b : Fortin et Soulié, 1983). La méthode des frontières (ou des équations intégrales) est généralement la mieux adaptée pour étudier le facteur de forme (Shahrour et al., 1996) car elle permet de placer la frontière latérale suffisamment loin de la cavité et de discrétiser uniquement le contour du domaine d'écoulement, ce qui permet de réduire considérablement le temps et le coût du calcul. Cette méthode a été utilisée pour étudier l'influence de l'extension latérale du domaine d'écoulement sur le facteur de forme. Les résultats obtenus sont portés sur la figure $3 \mathrm{~b}$. On peut constater que le facteur de forme $m$ croît sensiblement avec l'étendue du domaine défini par R/D avant de se stabiliser au voisinage de la valeur de $m$ donnée par Hvorslev (1951) pour une cavité considérée éloignée des limites de l'aquifère (rapports $\mathrm{H}_{w} / \mathrm{D}$ élevés). Ceci s'explique par le fait que la valeur du facteur de forme est donnée par Hvorslev dans un milieu infini alors que la présence d'une frontière imperméable (par exemple un obstacle tel que paroi, sous-sol enterré) à proximité de la cavité réduit le débit de percolation, et d'après l'équation (1), le facteur de forme.

Ces résultats donnent, en partie, une explication aux écarts notés entre les valeurs de m proposées par différents auteurs et montrent la nécessité de prendre en compte les conditions réelles de terrain (présence d'obstacles, niveau de la nappe, position du substratum) pour le calcul de m.

Aux difficultés d'exécution de l'essai et aux divergences de détermination de m s'ajoutent les sensibilités des méthodes d'interprétation pratique de l'essai Lefranc comme on peut le constater dans l'analyse des résultats d'essais réels qui suit.

\section{4}

\section{Méthodes d'interprétation pratique de l'essai Lefranc}

L'essai Lefranc peut être interprété par les méthodes suivantes (Norme NF P 94-132, AFNOR, 1992) qui dérivent toutes de l'application de la loi de Darcy :

- calage théorique sur les points expérimentaux de la charge hydraulique $\mathrm{h}$ ( $\mathrm{t}$ ) (équations 4 et 5 ) ;

- ajustement linéaire en coordonnées semi-logarithmiques (équation 6) ;

- interprétation numérique à partir de l'équation différentielle (équation 2).

\section{1}

\section{Calage théorique sur la courbe expérimentale de la charge hydraulique}

La courbe théorique de variation de la charge hydraulique h(t) donnée par les équations (4) et (5) peut être ajustée sur les points expérimentaux en faisant varier la valeur du coefficient de perméabilité $\mathrm{k}_{\text {. }}$. On peut alors fixer la valeur de $k_{1}$ qui conduit à un bon calage.

Dans les sols très peu perméables ( $k_{z}$ très faible), le calage des points expérimentaux peut se faire par une approximation linéaire de la courbe théorique à partir des équations (4) et (5). En effet, la variation de la charge $h(t)$ entre le début de l'essai $\left(t_{0}=0\right.$, charge initiale $h_{0}$ ) et le temps $t$ (charge $\left.h(t)\right)$ peut être, d'après la forme générale (4), exprimée par :

$$
h_{0}-h(t)=\left(h_{0}-h_{p}\right)\left(1-e^{-x}\right)
$$

avec :

$$
\mathrm{x}=\frac{\mathrm{m} \cdot \mathrm{k}_{\mathrm{L}} \cdot \mathrm{D}}{\mathrm{S}} \cdot \mathrm{t}
$$

Le développement limité d'ordre $1\left(x^{2}<<1\right.$ pour $k_{\text {, }}$ très faible) de la fonction exponentielle (équation $7 a$ ) conduit à une droite passant par l'origine d'équation :

$$
\mathrm{h}_{\mathrm{o}}-\mathrm{h}(\mathrm{t})=\left(\left(\mathrm{h}_{\mathrm{o}}-\mathrm{h}_{\mathrm{p}}\right) \cdot \mathrm{x}\right.
$$

et dont la pente $\alpha_{1}$ permet de calculer $k_{1}$, soit en introduisant (4b) : 


$$
\mathrm{k}_{\mathrm{L}}=\frac{\alpha_{1} \cdot \mathrm{S}+\mathrm{Q}_{\mathrm{a}}}{\mathrm{m} \cdot \mathrm{D} \cdot \mathrm{h}_{\mathrm{o}}}
$$

\section{2}

\section{Ajustement linéaire semi-logarithmique $\left(Q_{\theta}=0\right)$}

La procédure d'ajustement linéaire en coordonnées semi-logarithmiques peut être utilisée lorsque $Q_{a}=0$ (essai d'absorption ou de remontée d'eau dans le forage après pompage). En effet, en faisant intervenir les abaissements $\delta$ h dans le forage, l'équation (6) devient (RAT et Laviron, 1974; Chapuis, 1989 ; Cassan, 1980) :

$$
\mathrm{t}=-\frac{\mathrm{S}}{\mathrm{m} \cdot \mathrm{D} \cdot \mathrm{k}_{\mathrm{L}}} \operatorname{Ln} \frac{\mathrm{h}_{0}-\delta \mathrm{h}}{\mathrm{h}_{0}}
$$

Cette égalité peut être aussi obtenue par simple intégration de l'équation (2) pour $\mathrm{Q}_{\mathrm{a}}=0$ et les conditions initiales $\left(t=t_{0}=0, h=h_{0}\right)$.

Or, en traçant la droite expérimentale de la variation du temps t dans un diagramme en coordonnées semilogarithmiques, on peut déterminer la pente $\alpha_{2}$ de la courbe pour en déduire la valeur de $k_{\mathrm{L}}$, soit :

$$
\mathrm{k}_{\mathrm{L}}=-\frac{\mathrm{S}}{\mathrm{m} \cdot \mathrm{D} \cdot \alpha_{2}}
$$

avec :

$$
\alpha_{2}=\frac{\Delta t}{\Delta \operatorname{Ln}\left(\frac{h_{0}-\delta h}{h_{0}}\right)}
$$

\section{3}

\section{Interprétation numérique à partir de l'équation différentielle}

L'exploitation de l'essai Lefranc à partir de l'équation différentielle consiste à tracer la courbe représentative de la charge hydraulique $h(t)$ en fonction de la vitesse apparente de remontée ou de descente d'eau $\mathrm{dh} / \mathrm{dt}$ dans le forage. L'équation (2) montre que h(t) est une fonction linéaire de dh/dt. On peut alors construire l'allure des points expérimentaux $((\Delta h / \Delta t), h)$ avec :

et

$$
\begin{aligned}
& \left(\frac{\Delta h}{\Delta t}\right) j=\frac{h_{1}-h_{1-1}}{t_{1}-t_{i-1}}, \\
& h_{1}=\frac{1}{2}\left(h_{1}+h_{1-1}\right)
\end{aligned}
$$

Dans cette procédure d'exploitation, on peut distinguer aussi les deux cas :

a) $Q_{\mathrm{a}} \neq 0$, les points expérimentaux décrivent une droite passant par les deux points extrêmes :

$$
\begin{aligned}
& \cdot \frac{\Delta \mathrm{h}}{\Delta \mathrm{t}}=\frac{\mathrm{Qa}}{\mathrm{S}}, \mathrm{h}(\mathrm{t})=0, \\
& \cdot \frac{\Delta \mathrm{h}}{\Delta \mathrm{t}}=0, \quad \mathrm{~h}(\mathrm{t})=\frac{\mathrm{Qa}}{\mathrm{m} \cdot \mathrm{k}_{\mathrm{L}} \cdot \mathrm{D}}=\mathrm{h}_{\mathrm{a}},
\end{aligned}
$$

ce qui permet de déduire la valeur $\mathrm{k}_{\mathrm{L}}$, soit :

$$
\mathrm{k}_{\mathrm{L}}=\frac{\mathrm{Qa}}{\mathrm{m} \cdot \mathrm{D} \cdot \mathrm{h}_{\mathrm{a}}}
$$

On peut aussi, dans ce cas, déterminer la pente $\alpha_{3}$ de la droite de régression passant par les points les mieux alignés sur l'ensemble des points expérimentaux pour en déduire numériquement un coefficient de perméabilité à peu près constant, soit :

$$
\mathrm{k}_{\mathrm{L}}=\frac{\mathrm{S}}{\mathrm{m} \cdot \mathrm{D} \cdot \alpha_{3}}
$$

avec :

$$
\alpha_{3}=\frac{\Delta \mathrm{h}}{\Delta\left(\frac{\Delta \mathrm{h}}{\Delta \mathrm{t}}\right)}
$$

b) $Q_{n}=0$, la droite d'ajustement linéaire des points expérimentaux passe par l'origine et a pour pente $\alpha$. liée au coefficient de perméabilité $k_{L}$ par une égalité analogue à l'équation (10).

\section{5}

\section{Application à l'interprétation d'essais réels}

\section{1}

\section{Présentation des essais réalisés}

On exploite quatre essais réels effectués à charge variable (Fig. 2) sur des sols du bassin parisien de perméabilité différente :

- marno-calcaire de Brie altéré perméable :

$\mathrm{k}_{\mathrm{L}} \geq 10^{-5} \mathrm{~m} / \mathrm{s}$;

- sable fin de Fontainebleau et calcaire de Saint-Ouen peu perméable : $10^{-8}<\mathrm{k}_{\mathrm{L}}<10^{-5} \mathrm{~m} / \mathrm{s}$;

- marne bleue d'Argenteuil très peu perméable :

$\mathrm{k}_{\mathrm{i}}<10^{-8} \mathrm{~m} / \mathrm{s}$.

Les essais sont effectués à des profondeurs variant entre 5,0 et $16,5 \mathrm{~m}$ et avec des charges initiales $h_{\text {de }}$ d,0 à $16,5 \mathrm{~m}$. Dans chaque essai, la cavité (de 75 à $120 \mathrm{~mm}$ de diamètre) est isolée par un tubage $85 / 98 \mathrm{~mm}$ ou $120 / 140 \mathrm{~mm}$ avec la mise en place d'un gravier-filtre dans le marno-calcaire de Brie, dans les sables de Fontainebleau et dans le calcaire de Saint-Ouen résiduel (sol homogène, Fig. 2a) ainsi que dans les marnes bleues d'Argenteuil (sol stratifié, Fig. 2b). La longueur L de la cavité varie de $0,5 \mathrm{~m}$ à $2,0 \mathrm{~m}$, ce qui conduit à des rapports L/D de 4,2 à 26,7 et, d'après Hvorslev (Fig. 3a), à une valeur de $\mathrm{m}$ ( $=\mathrm{m}_{\sigma}$ Norme NF P 94-132, AFNOR, 1992) de 12,3 à 42,1. Dans le marnocalcaire de Brie altéré et les sables de Fontainebleau, l'essai est poursuivi après injection d'un début d'apport constant ( $Q$. non nul, voir Fig. 4 et 7 a) alors qu'il s'agit, dans le calcaire de Saint-Ouen et les marnes bleues d'Argenteuil, d'essais d'absorption $\left(Q_{\mathrm{a}}=0\right.$, Fig. 5, 6 et 7b).

\section{2}

\section{Calage théorique \\ sur les points expérimentaux de la charge hydraulique en fonction du temps}

Sur les figures 4 et 5 sont portés les points expérimentaux avec les courbes de calage théorique de la charge hydraulique $\mathrm{h}(\mathrm{t})$ en fonction du temps des quatre essais réalisés ; il ressort que : 
- dans les marno-calcaires de Brie (Fig. 4a), l'examen de l'allure des points expérimentaux montre qu'il subsiste, dès le début de l'essai, une légère stabilisation due probablement à un bourrage initial en début d'essai ; ensuite, il se produit en régime transitoire un léger colmatage entre environ 1000 et $1200 \mathrm{~s}$ avant d'atteindre le palier de stabilisation du régime permanent. Les deux courbes théoriques de calage (équations $4 \mathrm{a}$ et $4 \mathrm{~b}$ ) s'inscrivent plus ou moins bien dans les points expérimentaux et conduisent à des coefficients de perméabilité très proches $\left(\mathrm{k}_{\mathrm{t}}=1,2\right.$ et $\left.1,4 \cdot 10^{-5} \mathrm{~m} / \mathrm{s}\right)$;

- I'essai dans les sables de Fontainebleau (Fig. 4b) montre qu'un colmatage s'est produit avec débourrage dès le début de l'essai. Le calage sur les points expérimentaux ne permet pas de trouver des courbes théoriques (équation 4) qui s'ajustent au mieux sur l'ensemble des points obtenus et que les valeurs de $k_{1}$ de 1,$0 ; 1,5$ et $2,0.10^{-6} \mathrm{~m} / \mathrm{s}$ sont très peu influencées par le calage mais correspondent tout de même à un terrain totalement colmaté ;

- dans les essais d'absorption $\left(Q_{a}=0\right)$, les points expérimentaux de l'essai dans le calcaire de Saint-Ouen résiduel (Fig. 5a) décrivent une parfaite courbe du régime transitoire avant la stabilisation, en régime permanent, vers 1500 à $2000 \mathrm{~s}$. Le calage théorique (équation $7 \mathrm{a}, \mathrm{h}_{\mathrm{p}}=0$ ) conduit à $7,0.10^{-7} \mathrm{~m} / \mathrm{s}$ en début de l'essai et à $2,0.10^{-7} \mathrm{~m} / \mathrm{s}$ sur le palier de stabilisation, l'approximation linéaire (équation $7 \mathrm{~b}, \mathrm{Q}_{\mathrm{a}}=0$ ) à $6,0.10^{-7} \mathrm{~m} / \mathrm{s}$. En effet, il n'est pas possible de trouver dans l'essai une courbe théorique qui coincide avec l'ensemble des points expérimentaux. Mais, ce résultat, d'ordre purement mathématique, montre que l'approximation

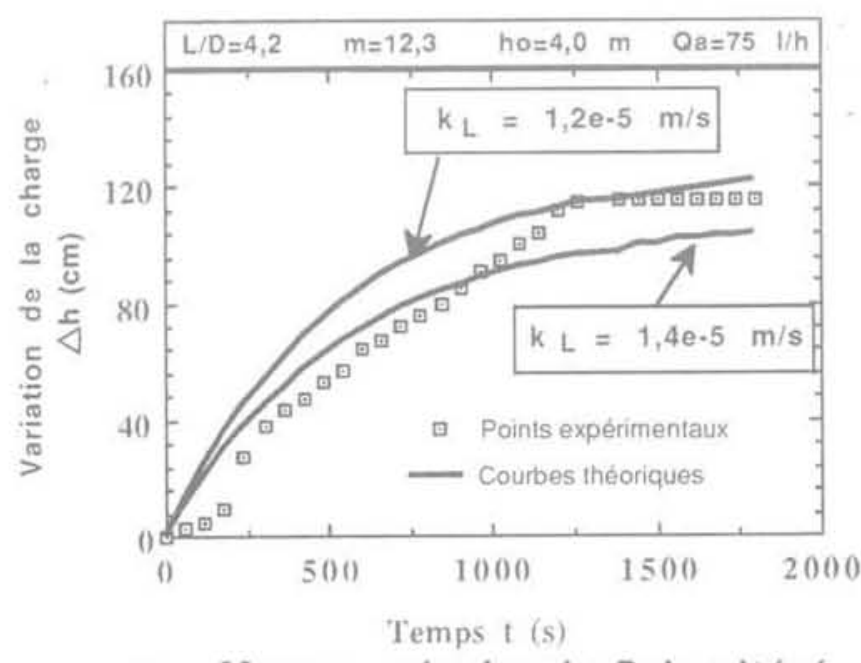

a - Marno-calcaire de Brie altéré

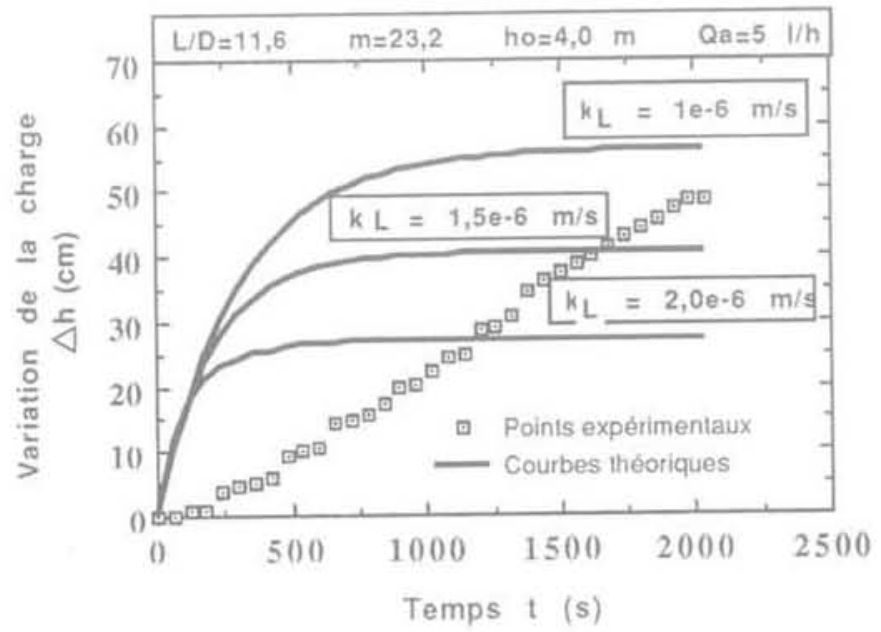

b - Sable fin de Fontainebleau

FIG.4 Calage sur la courbe expérimentale (charge hydraulique en fonction du temps).

Flow rate curve matching.

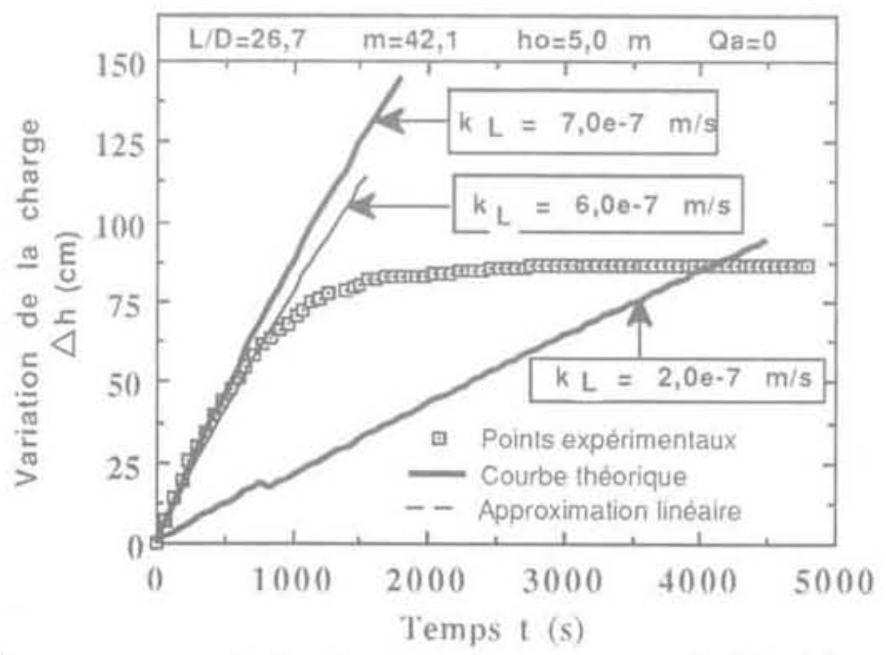

a - Calcaire de St-Ouen résiduel

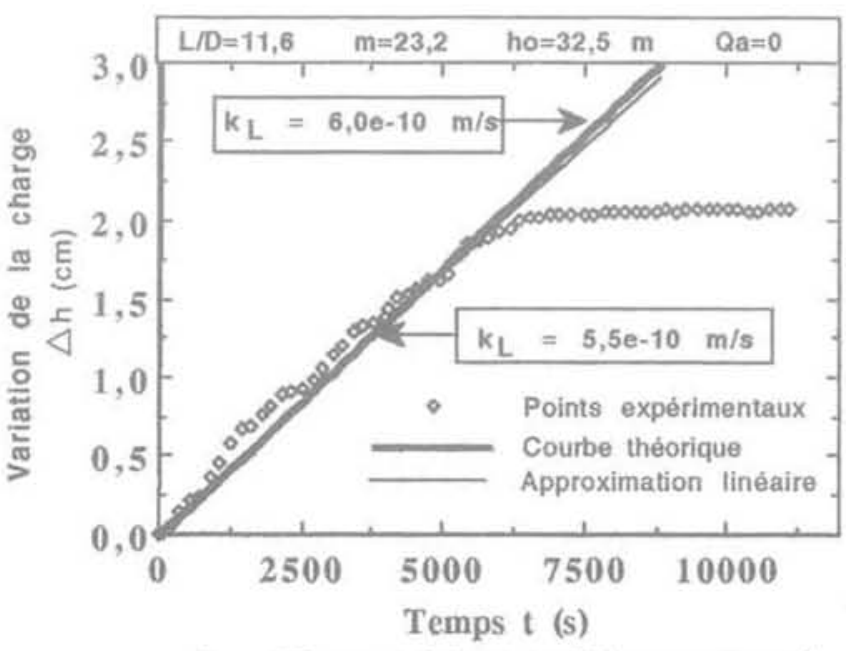

b - Marnebleue d'Argenteuil

FG.5 Calage sur la courbe expérimentale de la charge hydraulique en fonction du temps.

Flow rate curve matching. 
linéaire proposée (équation 7 b) peut être parfaitement retenue dans l'interprétation de l'essai dans les sols peu à très peu perméables (généralement lorsque $k$. est inférieur à $10^{-7} \mathrm{~m} / \mathrm{s}$ ). En effet, les deux valeurs de 6,0 et $7,0.10^{-7} \mathrm{~m} / \mathrm{s}$ sont confirmées par les autres méthodes d'interprétation présentées dans le paragraphe qui suit.

Dans les marnes bleues d'Argenteuil du Ludien (Fig. 5b), le régime permanent et atteint vers $6200 \mathrm{~s}$ avec de légères perturbations (colmatage et débourrage) dans la phase transitoire. Le calage théorique (forme exponentielle, équation $7 \mathrm{a}$ avec $\mathrm{h}_{\mathrm{p}}=0$ ) sur les points expérimentaux et l'approximation linéaire (équation $7 c, Q_{a}=0$ ) conduisent à des valeurs de $k_{L}$ très proches et qui sont respectivement de $6,0,10^{-10} \mathrm{~m} / \mathrm{s}$ et de $5,5.10^{-10} \mathrm{~m} / \mathrm{s}$.

Le calage sur la courbe expérimentale de la charge hydraulique en fonction du temps montre que le coefficient de perméabilité $k$, peut être évalué avec des valeurs dans un rapport allant de 1 à 4 , ce qui est normal pour la mesure de la perméabilité en place des sols.

\section{3}

\section{Ajustement linéaire en coordonnées semi-logarithmiques $\left(Q_{a}=0\right)$}

Les résultats des essais d'absorption sur le calcaire de Saint-Ouen résiduel et les marnes bleues sont reproduits dans les diagrammes en coordonnées semi-logarithmiques de la figure 6 . Ces graphiques montrent, dans le cas du calcaire de Saint-Ouen résiduel (Fig. 6a), que l'ajustement linéaire ne peut être fait que sur la première partie linéaire de la courbe semi-logarithmique et permet d'avoir $\mathrm{k}_{1}$ égal à $6,5 \cdot 10^{-7} \mathrm{~m} / \mathrm{s}$ qui concorde avec la valeur de $7,0.10^{-7} \mathrm{~m} / \mathrm{s}$ obtenue par le calage sur les points expérimentaux de la charge hydraulique (Fig. 5a). L'ajustement sur le palier de stabilisation conduit à une valeur plus faible, soit $2,4.10^{-8}$ $\mathrm{m} / \mathrm{s}$ correspondant au régime permanent.

Dans les marnes bleues d'Argenteuil, la valeur du coefficient de perméabilité de $5,7.10^{-10} \mathrm{~m} / \mathrm{s}$ fixẻe par l'ajustement linéaire (Fig. 6b) s'inscrit dans la plage de variation de $\mathrm{k}$, obtenue par le calage sur la courbe expérimentale (Fig. 5b). L'ajustement sur le palier de stabilisation du régime permanent conduit à $2,0.10^{-10} \mathrm{~m} / \mathrm{s}$.

Dans les deux essais d'absorption, l'ajustement linéaire en coordonnées semi-logarithmiques effectué sur le palier de stabilisation du régime permanent conduit à des valeurs de $k_{\mathrm{L}}$ de 2 à 3 fois plus faibles que celles données par le régime transitoire.

On peut constater ici que la valeur de $k$, peut être estimée dans une fourchette de valeurs de 1 à 3 .

\section{4}

\section{Interprétation numérique à partir de l'équation différentielle}

La construction des courbes représentatives de la charge hydraulique $h(t)$ en fonction de dh/dt montre qu'il est possible de fixer, dans les marno-calcaires de Brie altéré (Fig. 7a), deux zones distinctes où les points expérimentaux sont peu alignés ; mais on peut déterminer deux coefficients de perméabilité légèrement différents qui sont de $1,0,10^{-5} \mathrm{~m} / \mathrm{s}$ et $7.9 .10^{-6} \mathrm{~m} / \mathrm{s}$. Les résultats de l'essai dans le calcaire de Saint-Ouen résiduel (Fig. 7b) permettent de distinguer deux zones à peu près linéaires avec $k_{1}$ de $6,7.10^{-7} \mathrm{~m} / \mathrm{s}$ dans la phase du régime transitoire et de $3,3.10^{-7} \mathrm{~m} / \mathrm{s}$ en régime permanent (phase de stabilisation).

Par contre, dans les sables de Fontainebleau, il y a une très forte dispersion des points expérimentaux; cette technique d'exploitation doit être éliminée et la prudence s'impose car les coefficients de perméabilité obtenus par le calage sur la courbe expérimentale de la charge hydraulique en fonction du temps correspondent à un terrain colmaté dès le début de l'essai; les valeurs « réelles » de $k_{\mathrm{L}}$ sont naturellement plus élevées. Il n'y a pas, à ce jour, des termes correcteurs contrairement au phénomène de remontées du sable dans le forage (cas des cavités de faible hauteur) où le coefficient de perméabilité $k_{1}$ est évalué à la hausse à partir des abaques de Soletanche (Cassan, 1980).

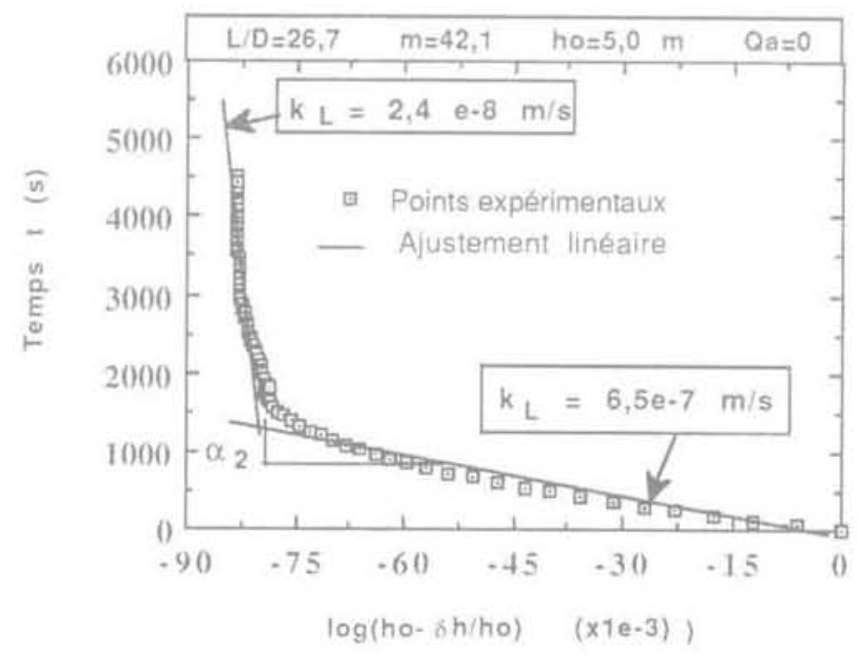

a - Calcaire de St-Ouen résiduel

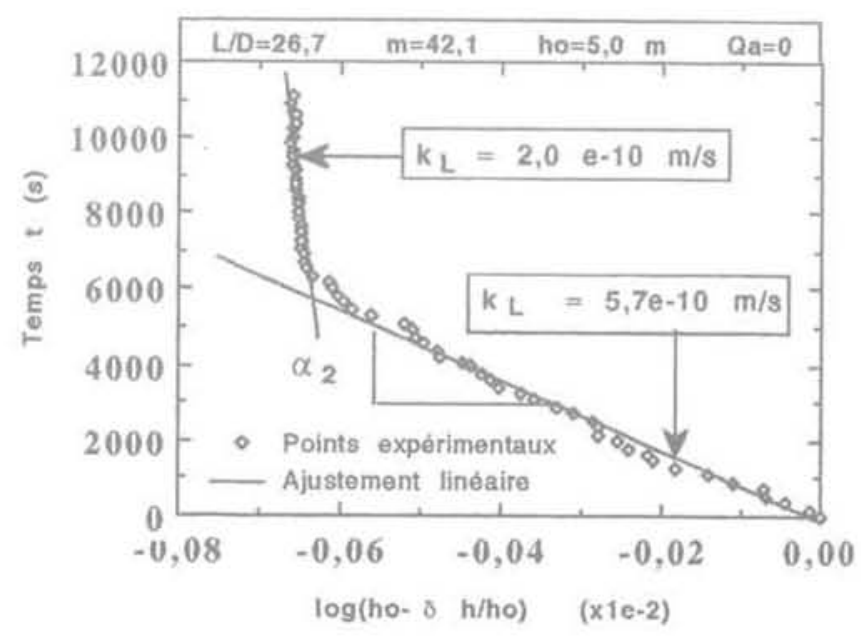

b - Marne bleue d'Argenteuil

FiG.6 Calage en coordonnées semi-logarithmiques. Matching on semi-logarithmic co-ordinates. 


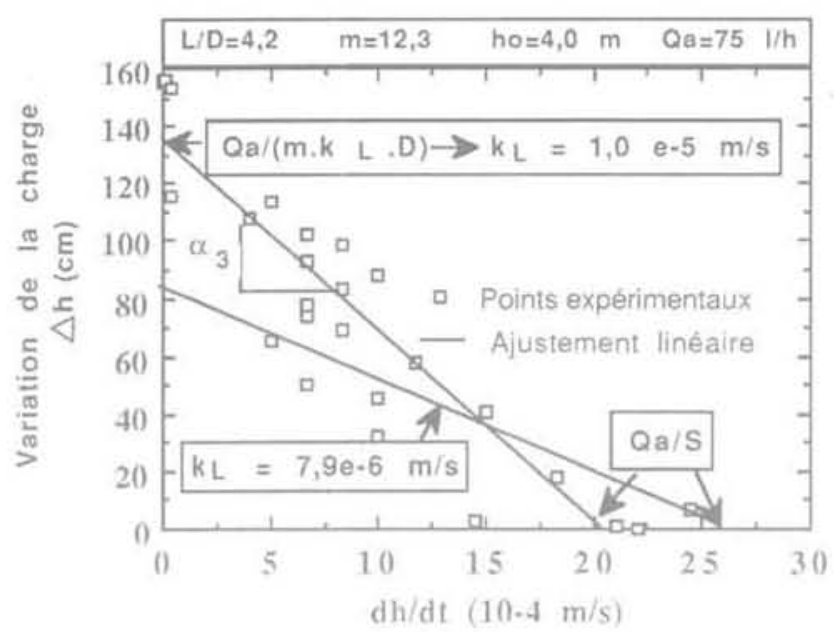

a - Marno-calcaire de Brie Altéré

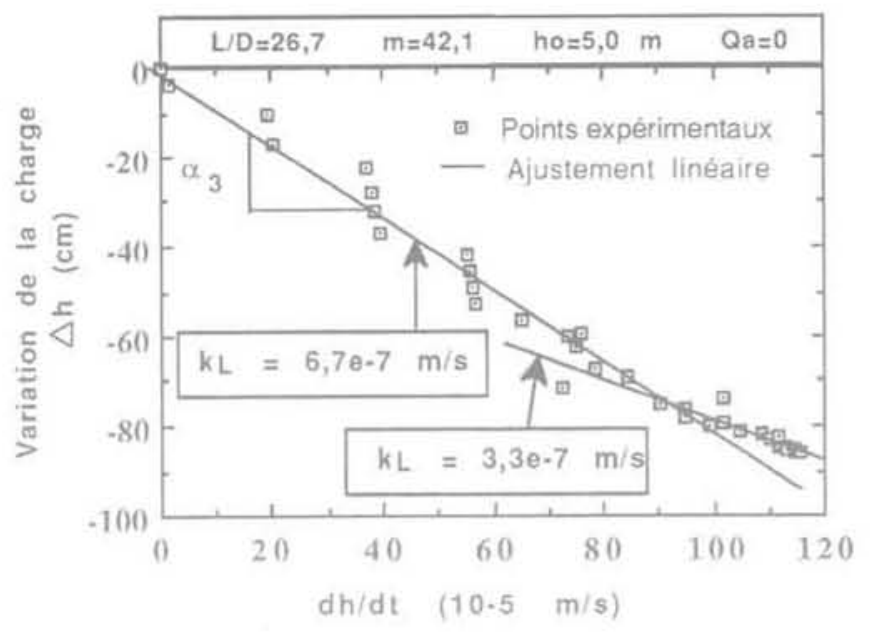

b - Calcaire de St-Ouen

FG. Interprétation à partir de l'équation différentielle. Interpretation based on the differential equation.

Dans les marmes bleues d'Argenteuil, on constate le mếme phénomène de dispersion des points expérimentaux; l'exploitation de l'essai à partir de cette méthode est aussi à éliminer : les valeurs de $k$. obtenues par les deux techniques d'interprétation précédentes (Fig. $5 \mathrm{~b}$ et $6 \mathrm{~b}$ ) concordent entre elles; celles-ci peuvent ètre retenues.

L'exploitation numérique de l'essai Lefranc à partir de l'équation différentielle permet, si l'essai est « exploitable $»$, de déterminer ici les valeurs de $k$. dans une fourchette de 1 à 3 . La recherche d'un ajustement sur les points peu alignés peut conduire à des coefficients de perméabilité $k_{1}$ très dispersés avec un rapport de 1 à 1000 (Rat et Laviron, 1974). Pour ces raisons, la méthode d'interprétation de l'essai Lefranc à partir de l'équation différentielle doit être automatiquement éllminée dès que les points de mesure ne présentent pas de zones où ils peuvent être alignés.

\section{5}

\section{Recommandations relatives à l'interprétation de l'essai}

L'interprétation pratique de l'essai Lefranc nécessite un balayage des résultats expérimentaux le plus large possible par les différentes techniques d'exploitation. Sur le plan pratique, une interprétation rigoureuse de l'essai doit respecter les étapes suivantes:

1) Porter les points expérimentaux sur un graphique de la charge hydraulique h(t) en fonction du temps et procéder à un calage théorique (équations 4, 5 et 7).

2) Construire les courbes représentatives de la charge hydraulique $h(t)$ en fonction de la vitesse apparente de remontée ou de descente $\mathrm{dh} / \mathrm{dt}$ dans le forage, et chercher les zones où les points expérimentaux constituent une droite ou plusieurs droites pour déterminer une ou plusieurs valeurs de $k_{\text {f }}$ (équations 9 et 10). A travers plusieurs essais, nous avons constaté que cette méthode conduit généralement à des résultats ( inexploitables » dès qu'apparaissent des phénomènes de colmatage et de débourrage même si l'interprétation de l'essai est possible par les autres méthodes;
3) Dans les essais d'absorption $\left(Q_{a}=0\right)$, porter les points expérimentaux dans un plan èn coordonnées semi-logarithmiques (équation 8). Cette technique d'ajustement linéaire aboutit généralement à des résultats « exploitables ».

L'ensemble des méthodes d'interprétation nécessite un nombre important de points expérimentaux, ce qui dernande un temps d'essai relativement long et un pas de mesure réduit (en particulier dans les matériaux peu perméables) afin de mieux appréhender le calage et les ajustements linéaires et de déceler toutes les anomalies possibles (colmatage, débourrage). Toute méthode qui conduit à de fortes dispersions des points expérimentaux doit être automatiquement éliminée.

4) Calculer le facteur de forme m. Les nombreuses approches existantes conduisent à de réelles discordances et montrent que l'évaluation de m aboutit à des écarts qui peuvent être très importants. Cependant, une approximation de m par Hvorslev (1951) nous semble raisonnable à condition de prendre en compte les conditions aux limites en particulier la position de la nappe et du substratum imperméable (Cassan, 1980).

5) Comparer les valeurs du coefficient de perméabilité obtenues en éliminant celles qui paraissent peu "représentatives » du matériau d'essai. Là, la prudence et l'examen critique des résultats s'imposent avant de procéder au maintien ou à l'élimination d'une valeur par rapport à une autre. Dans les quatre exemples d'essais interprétés, il est clair que les valeurs de perméabilité de 1,0 à $2,0.10^{-6} \mathrm{~m} / \mathrm{s}$ obtenues dans les sables de Fontainebleau sont à éliminer (terrain colmaté) : par contre des valeurs moyennes peuvent être retenues dans les autres cas, soit $1,0.10^{-5} \mathrm{~m} / \mathrm{s}$ dans le marno-calcaire de Brie altéré, $6,5.10^{-7} \mathrm{~m} / \mathrm{s}$ dans le calcaire de Saint-Ouen résiduel et $5,0.10^{-10} \mathrm{~m} / \mathrm{s}$ dans les marnes bleues d'Argenteuil. Par ailleurs, à l'exception de l'essai dans les sables de Fontainebleau, les trois essais montrent que la valeur du coefficient de perméabilité calculée dans la phase du régime transitoire est de 2 à 3 fois plus élevée que celle de la phase du régime permanent calculée sur le palier de stabilisation. 


\section{Conclusion}

La présente étude montre que la mesure du coefficient de perméabilité locale k, par l'essai Lefranc conjugue difficultés expérimentales, divergences des approches du calcul du facteur de forme m et sensibilités des méthodes d'interprétation.

Sur le plan expérimental, l'essai Lefranc nécessite des dispositions particulières pour réduire les sources d'erreurs: connaissance précise de la position de l'aquifère, exécution soignée (sans boue) de la cavité pour éviter le colmatage préalable, isolation totale de la poche pour éviter toutes les fuites et les communications de nappes, curage de la cavité afin d'éliminer les particules qui peuvent entraîner et accélérer le colmatage, stabilisation des parois de la lanterne par un gravier-filtre, suivi précis et suffisamment long de la variation de la charge en particulier dans le cas des sols peu perméables (essais d'absorption).

L'interprétation pratique de l'essai Lefranc demande un large balayage des points expérimentaux par toutes les techniques d'exploitation possibles : calage des points expérimentaux sur la courbe de la charge hydraulique en fonction du temps, ajustement linéaire en fonction de la vitesse apparente de remontée ou de descente d'eau et en coordonnées semi-logarithmiques $\left(Q_{0}=0\right)$. Chaque méthode d'interprétation demande un nombre important de points de mesure pour pouvoir vérifier la validité de l'essai (anomalies en cours d'essai, précision du calage et de l'ajustement linéaire),

En effet, aux incertitudes sur le plan expérimental s'ajoute le choix d'une méthode d'interprétation qui peut conduire, en particulier lorsque l'essai est mal exécuté, à de fortes dispersions.

Face à ces difficultés, une bonne estimation du coefficient de perméabilité Lefranc passe tout d'abord par une exécution soignée de la cavité et une amélioration des moyens de suivi et de contrôle de l'essai ; à cela, il convient d'associer un nombre important de points expérimentaux pour assurer une meilleure interprétation possible. Mais, quelles que soient les dispositions prises, il ne faut pas oublier que l'essai Lefranc est ponctuel et qu'il faut en multiplier le nombre à chaque projet en fonction des objectifs recherchés.

\section{REMERCIEMTENTS}

Les auteurs tiennent â remercier vivement MM. Pierre Habib et Michel Gambin qui ont enrichi la présente communication par leurs remarques et leur conseil pour formuler une version moins critique vis-â-vis de l'essai Lefranc.

\section{Bibliographie}

AFNOR, Norme Française NF P 94-132. Sols : Reconnaissance et essais, Essais d'eau Lefranc, juin 1992, 16 p.

AFNOR, Norme Française NF P 94-090, Mesure de la perméabilité en laboratoire. 1992

Bjerrum L. Nash J.K.T.L. Kennard R.M. Gibson R.E. - \& Hydraulic fracturing in field permeability testing $x$. Géotechnique, vol. 22, $n^{2}$ 2, 1972, p. 319-322

Boersma L. - «Field measurement of hydraulic conductivity below a water table D. Methods of Soil Analysis, Part 1. Éditions C.A. Black. American Society of Agronomy, Madison (W1), 1965 p. $222-233$

Bouwer H. Jackson R.D. - « Determining soil propertie n. Drainage for Agricutture, Edition Schilfgaarde, American Society of Agronomy, Madison (WI). 1974, p. 611-672.

Cassan M - «Filtrations dans les cavités souterraines : applications á l'épuisement des fouilles superficielles, Première partie: Cavités sphériques y. Annales de ITTBTP, $n^{\circ} 371$, avril 1979, $51 \mathrm{p}$.

Cassan M. - Les essais d'eau dans la reconnaissance des sols. Eyrolles Ch. III, 1980, p. 39-72.

Chapuis R.P. - $\propto$ Shape factors for permeability tests in boreholes and piezometer. $)$ GroundWater, vol. 27. $n^{\circ} 5,1989$, p. 647-654.

Dachler R. - Grundwasserstromung (Flow of Ground Water). Wien, Julius Springer, 1936, $141 \mathrm{p}$.

Fortin M. Soulié M. - « A non-conforming piecewise quadratic finite element on

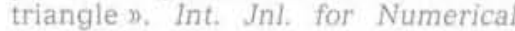

Methods in Engineering, vol. $19, \pi^{\circ} 4$ 1983 , p. 505-520.

Gibson R.E. - «An analysis of system flexibility and its effect on time lag in porewater measurement 1. Geotechnique. vol. $13, n^{\circ} 1,1963$, p. $1-11$

Gibson R.E. - $\alpha$ A note on the constant head test to measure soil permeability in situ n. Geotechnique, vol. 16, n³, 1966 p. 256-257.

Hvorslev M.J. - «Time lag and soil per. meability in ground water levels and pressures w. US Army Engineer Waterways Experiment Station, MS. Bulletin 36, 1951.

Kallstenius T., Wallgren A. - $\alpha$ Pore pressure measurements in field investiga tions v. Proc. of the Royal Swedish Geotechnical Inst. $\mathrm{n}^{\mathrm{D}} 13,1956$.

Lefranc E. - "Procédé de mesure de la perméabilité des sols dans les nappes aquifères et application au calcul di débit des puits $\mathrm{D}$. Le Génie Civil, CIX, n 15, 1934, p. 306-308

Lefranc E, - a La théorie des poches absorbantes et son application à la détermipation du coefficient de perméabilité en place et au calcul du débit des nappes d'eau ». Le Génie Civil, CXI. n²0, 1937 . p. $409-413$.

MELT. - Fascicule 62, Titre V, Éditions de la direction des Journaux officiels, 1993 $182 \mathrm{p}$.

Milligan V. - « Field measurement of permeability in soil and rock n. Proc. ASCE Conf. on In Situ Measurement of Soils Properties, Raleigh, NC., vol. 2, 1975, p. 3-36.

Randolph M.F.. Booker J.R، - a Analysis of seepage into a cylindrical permeame- ter. $n$ Proc, of the 4 th Int. Conf. on Num. Methods in Geomechanics, Edmonton. vol. 1, 1982, p. 349-357.

Rat M. Laviron F. - u Mesure du coefficient de perméabilité par essais ponctuels $\gg .2^{e}$ Congrès international de Géologie de l'ingénieur, Sao Paulo, août 1974. Bull. Liaison Labo. P. et Ch., sept.oct. 1974, p. 179-182.

Raymond G.P., Azzouz M.M. - « Permeabllity determination for proceding rates of consolidation 1. Institution of Civil Engineers, Londion, 1969, p. 285-293.

Schneebeli G. - «esure in situ de la perméabilité d'un terrain $\gg$. Comptes rendus des $3^{\text {ts }}$ Journées d'hydraulique, Alger, 1954. p. 270-279.

Schneebeli G. - Hydraulique souterraine, Paris, Evrolles, 1966, 362 p.

Shahrour I, Lafhaj Z. Dhoulb A. - «Étude numérique du coefficient de forme utilisé dans l'interprétation des essais in situ pour la mesure de la perméabilité des sols n. Colloque du Réseau ces laboratoires GEO, Aussois, décembre 1996.

Tavenas F. Tremblay M. Larouche G. Leroueil S. - - In situ measurement of permeability in soft clays n. Proc., ASCE Speciality Conf. in situ. 86, Blacksburg (VA), 1986, p. 1034-1048.

Tavenas F., Diene M., Leroueil S, - « AnaIysis of in situ constant head permeability test $\$$. Proc., 39th Canadian geotechnical Conf. Ottawa, 1986, p. 71-77.

Weber W.G. - $*$ In situ permeabilities for determining rates of consolidation n. Highway Research Record, vol. 243, 1968, p. 49-61.

Wilkinson W. B. - "Constant head in situ permeability tests in clay strata n. Géotechnique, vol. $18, n^{\circ} 2,1968$, p. 172-194. 